\title{
Enseigner à l'école primaire une géographie problématisée : un défi?
}

\section{Teaching a Problematized Geography in Elementary School: a Challenge? \\ Enseñar en la escuela primaria una geografía problematizada: un desafío?}

\section{Thierry Philippot}

Volume 15, numéro 1, 2012

La problématisation des apprentissages en enseignement de l’histoire-géographie : quels cadres de référence, quels fondements?

URI : https://id.erudit.org/iderudit/1013377ar

DOI : https://doi.org/10.7202/1013377ar

Aller au sommaire du numéro

Éditeur(s)

Faculté d'éducation, Université de Sherbrooke

ISSN

1911-8805 (numérique)

Découvrir la revue

Citer cet article

Philippot, T. (2012). Enseigner à l'école primaire une géographie problématisée : un défi? Nouveaux cahiers de la recherche en éducation, 15(1), 21-34. https://doi.org/10.7202/1013377ar

\section{Résumé de l'article}

Depuis la fin des années 1980, les didacticiens de la géographie et les textes officiels mettent en avant un enseignement problématisé de la géographie. Pour les enseignants généralistes de l'école primaire française, cette problématisation est-elle aussi évidente que cela à mettre en oeuvre dans leurs pratiques d'enseignement? Après avoir précisé le sens des termes «problème» et "problématisation» dans le champ scolaire, nous présentons ces propositions didactiques adressées aux enseignants. Enfin, nous mobilisons les résultats d'une recherche empirique qui donne à voir une géographie enseignée peu "problématisée», ce qui conduit à penser que la «problématisation» dans l'enseignement de la géographie à l'école primaire ne semble pas aller de soi. 


\title{
Enseigner à l'école primaire une géographie problématisée: un défi?
}

\author{
Thierry Philippot \\ Université de Reims Champagne-Ardenne IUFM
}

\section{Résumé}

Depuis la fin des années 1980, les didacticiens de la géographie et les textes officiels mettent en avant un enseignement problématisé de la géographie. Pour les enseignants généralistes de l'école primaire française, cette problématisation est-elle aussi évidente que cela à mettre en œuvre dans leurs pratiques d'enseignement? Après avoir précisé le sens des termes «problème» et «problématisation» dans le champ scolaire, nous présentons ces propositions didactiques adressées aux enseignants. Enfin, nous mobilisons les résultats d'une recherche empirique qui donne à voir une géographie enseignée peu «problématisée», ce qui conduit à penser que la «problématisation» dans l'enseignement de la géographie à l'école primaire ne semble pas aller de soi.

Mots-clés: géographie scolaire, école primaire, problématisation, didactique, travail enseignant

\section{Teaching a Problematized Geography in Elementary School: a Challenge?}

\section{Abstract}

Since the late 1980s, geography didacticians and standard texts have presented problematization in geography teaching. Is this problematization easy to actualize in the teaching practices of generalist teachers in French elementary schools? After defining the terms "problem" and "problematization" in the context of classroom teaching, we offer teachers didactical suggestions for their use. We then analyse results of empirical research that sheds light on teaching geography with little "problematization." This leads to the observation that "problematization" in geography teaching in elementary schools is not necessarily always present.

Key words: school geography, elementary school, problematization, didactics, teaching

\section{Enseñar en la escuela primaria una geografía problematizada: un desafío?}

\section{Resumen}

Desde finales de los años 1980, los didácticos de la geografía y los textos oficiales anteponen una enseñanza poco problematizada de la geografía. ¿Qué tan fácil está para los docentes generalistas de la escuela primaria francesa poner en práctica esta problematización en su enseñanza? Tras precisar el sentido de los términos «problema» y «problematización» en el campo escolar, presentamos propuestas didácticas dirigidas a los docentes. Por terminar, analizamos los resultados de una investigación empírica que deja entrever una geografía enseñada poco «problematizada», lo que lleva a pensar que «la problematización» en enseñanza de la geografía en la escuela primaria no parece ser evidente.

Palabras clave: geografía escolar, escuela primaria, problematización, didáctica, trabajo docente 


\section{Introduction}

En France, «la problématisation est à la mode» (Orange, 2005, p. 69) et le paradigme du problème se généralise dans le champ de l'enseignement (Fabre, 1997, 2005a; Rey, 2005). Cette généralisation du problème et cette injonction à problématiser (Benoît, 2007; Fabre, 2005b) s'inscrivent dans un contexte plus général marqué par la mise en avant dans les discours pédagogiques, des pédagogies actives et de l'importance de la motivation des élèves pour favoriser les apprentissages. Elles se trouvent confortées par les discours didactiques, inscrits dans le paradigme constructiviste, qui promeuvent la construction des savoirs en lieu et place de leur transmission dans une logique cumulative.

Dans ce contexte, les écrits de didacticiens de la géographie et les textes institutionnels diffusent l'image d'une géographie à enseigner problématisée. Une forme de consensus semble exister aujourd'hui sur ce vers quoi doivent tendre la géographie et son enseignement à l'école primaire. Ces écrits de diverse nature peuvent être considérés comme des prescriptions (Daniellou, 2002) définissant ce que l'enseignant devrait faire dans sa classe, sans pour autant préciser comment ce dernier pourrait le faire. Or, les travaux portant sur le travail enseignant (Saujat, Amigues et Faïta, 2007; Tardif et Lessard, 1999) insistent sur la distance entre travail prescrit et travail réel. Dès lors, on ne peut que s'interroger sur ce qui se joue dans les classes lorsque des enseignants enseignent de la géographie. Ce consensus apparent à propos de la géographie à enseigner s'étend-il aux pratiques d'enseignement ou celles-ci sont-elles en décalage, voire en rupture, par rapport à ces prescriptions auxquelles doivent faire face les enseignants généralistes de l'école primaire?

Bien que d'un usage fréquent dans le champ de l'enseignement, les termes «problème» et «problématisation»n'en demeurent pas moins polysémiques au point de relever parfois dans les discours du sens commun pédagogique (Astolfi, 2008). Il importe donc dans un premier temps de tenter de préciser le sens que ces notions peuvent prendre dans le domaine scolaire. Dans un second temps, nous présentons les différentes prescriptions adressées aujourd'hui aux enseignants de l'école primaire. Enfin, nous mobilisons les résultats d'une recherche empirique qui prend comme objet d'analyse des pratiques d'enseignement de la géographie à l'école primaire (Philippot, 2008) Les résultats obtenus donnent à voir une géographie peu problématisée, expression des difficultés d'enseignants généralistes à enseigner une discipline dont ils n'ont pas une image précise de l'objet, des démarches et des finalités. Autant d'éléments qui font que la problématisation dans l'enseignement de la géographie à l'école primaire ne semble pas aller de soi.

\section{Problématisation/problème, un champ sémantique polysémique}

Plusieurs auteurs (Astolfi, 2008; Orange, 2005; Partoune, 2002) soulignent le caractère polysémique de ces deux termes souvent associés tant dans l'enseignement universitaire que dans l'enseignement scolaire.

Le mot «problème» est défini, dans un premier sens, comme «une question à résoudre qui prête à discussion dans une science» (Dictionnaire Le Robert, 1992 p. 1534). Dans un second sens, c'est «une difficulté qu'il faut résoudre pour obtenir un certain résultat» (Ibid., p. 1534). Dans les deux sens, le problème nécessite la mobilisation d'un sujet et de ses capacités intellectuelles, 
mais dans le premier, la perspective est épistémologique, alors que dans le second, elle est plus d'ordre psychologique; le problème est une difficulté rencontrée. Quant au mot «problématisation», il renvoie à la notion de problématique, «art, science de poser les problèmes» (Ibid., p. 1534), et peut donc être considéré comme la capacité intellectuelle pour un sujet à construire un problème. Ce rapide détour par les significations de ces termes conduit à proposer une distinction entre construire un problème et résoudre un problème.

Dans le système éducatif, il apparaît nécessaire de distinguer ces termes et leurs usages dans les disciplines scientifiques et dans le champ scolaire. En effet, dans les disciplines scientifiques la problématisation, entendue comme construction du problème, a une fonction épistémique. La problématisation s'inscrit alors dans un paradigme scientifique et participe au processus de production de nouveaux savoirs par le chercheur. Dans cette perspective le problème est problème de recherche, il est construit par le chercheur, «dans la vie scientifique, les problèmes ne se posent pas d'eux-mêmes. [...]. S'il n'y a pas eu de questions, il ne peut y avoir connaissance scientifique» (Bachelard, 1970, p. 16).

L'école et le monde de la recherche ne poursuivent pas les mêmes finalités; l'enseignant pas plus que les élèves ne sont des chercheurs. De ce fait, il ne peut y avoir homologie entre les significations accordées à «problème» et «problématisation» dans ces deux mondes, même si des proximités sémantiques sont possibles. Se pose alors la question du passage d'un monde à l'autre de ces termes et de leurs usages. Ainsi, l'une des finalités de l'école est la transmission de savoirs scolaires. Or, par nature, ces savoirs sont des savoirs «décontextualisés par rapport à leur instance de production originelle» (Lautier, 2006, p. 73). Dès lors, problématiser peut être considéré comme une démarche conduite par l'enseignant dans le but de redonner du sens aux apprentissages dans les disciplines scolaires et aussi de motiver les élèves en leur proposant de résoudre un problème. Problématiser dans le monde scolaire apparaît comme une proposition alternative adressée aux enseignants, il s'agit pour eux «de problématiser le savoir scolaire, de transformer des constats et des résultats en questions, en interrogations, pour donner du sens à un cours qui ne se veut plus simple discours expositif» (Le Roux, 2004, p. 25). La fonction du problème est alors différente; il n'est plus question de produire des connaissances. Dans le monde scolaire, la particularité du problème «est d'exister pour produire un apprentissage» (Audigier, 1991, p. 17). On comprend donc qu'à «l'école le mot problème n'a pas la même signification qu'ailleurs» (Astolfi, 2008, p. 99). C'est une fiction sous contrôle (Partoune, 2002) de l'enseignant qui doit permettre aux élèves d'apprendre. De même, l'acte de problématiser doit être considéré différemment selon que l'on se situe dans le monde scientifique ou dans le monde scolaire. Dans ce dernier, apprendre à construire un problème est alors une démarche intellectuelle qui peut contribuer à la formation de l'esprit scientifique des élèves (Cariou, 2009), au développement de leur pensée (Demers, Lefrançois et Éthier, 2010) et donc constituer un objectif d'apprentissage. Une transposition du problème et de la problématisation du monde scientifique au monde scolaire est possible. Toutefois, du point de vue de l'enseignant, cela suppose alors qu'il soit en mesure de construire le problème et/ou de guider les élèves dans l'apprentissage du processus de problématisation, mais aussi de le contrôler en situation d'enseignement. Dans tous les cas, cela nécessite une réflexion sur les contenus à enseigner, une réflexion sur le «à partir de quoi» problématiser et une maîtrise de l'épistémologie de la discipline enseignée. Pour les élèves, l'approche par problème est présentée aujourd'hui comme devant leur permettre de construire les savoirs, de développer des capacités intellectuelles de l'ordre de l'analyse, de la mise en relation, de l'argumentation, dans le cadre de raisonnements. 
Confronter les élèves à un problème, c'est les confronter à «une tâche difficile qui se distingue d'une activité de simple exécution et qui exige l'invention d'une solution; il s'agit également d'une difficulté objective concernant un savoir ou savoir-faire; c'est enfin une tâche complexe où plusieurs compétences sont en jeu et qui se distingue d'un exercice ciblé» (Fabre, 1997, p. 50).

\section{Des propositions nouvelles pour l'enseignement de la géographie à l'école élémentaire}

Dans le même temps où le problème s'étend à l'ensemble du système éducatif, se développent des propositions nouvelles pour l'enseignement de la géographie à l'école primaire. Ces propositions émanent, pour l'essentiel, de deux origines différentes, la didactique des chercheurs et les textes institutionnels (programmes d'enseignement, rapport de l'Inspection générale).

\subsection{La didactique des chercheurs}

Depuis vingt ans, «l'idée de problème occupe fortement le paysage didactique» (Audigier, 1991, p.17). Des travaux en didactique de la géographie (Baldner, Clary et Élissalde, 1995; Bouchut, 2006; Considère, 2000; Gérin-Grataloup, Solonel et Tutiaux-Guillon, 1996; Le Roux 1997; Masson, 1994) proposent des démarches didactiques susceptibles de renouveler l'enseignement de cette discipline. Fortement marqués par le paradigme constructiviste, ces travaux promeuvent un enseignement problématisé de la géographie «qui devrait permettre de familiariser les jeunes avec le monde dans lequel ils vivent, de les amener à une représentation scientifique en leur fournissant l'outillage conceptuel et méthodologique indispensable à qui veut comprendre territoires et sociétés» (Baldner et al., 1995, p. 41). Cet enseignement doit se faire «en privilégiant les situationsproblèmes» (Ibid., p. 91). Le problème est donc au cœur des dispositifs didactiques que l'enseignant devrait construire dans le but que les élèves apprennent. Le problème devient un mot d'ordre en didactique «il faut des problèmes, il faut donner aux élèves des problèmes à résoudre» (Le Roux, 1997, p. 151). Si pour Le Roux le problème est construit et proposé par l'enseignant, il incombe à l'élève de le résoudre, "pour comprendre, apprendre et prouver qu'il a compris, l'élève doit "résoudre un problème", c'est-à-dire qu'il est contraint à raisonner sur des phénomènes» (Ibid., p. 152). C'est à partir de problèmes à construire ou à résoudre que l'on envisage le développement des connaissances et compétences chez les élèves, «la géographie se doit de proposer à l'élève une démarche qui lui permette de construire un questionnement (souvent à partir de cas particuliers observés), de mobiliser des connaissances puis d'en acquérir de nouvelles, d'identifier les relations et interrelations qui régissent certains aspects du phénomène géographique étudié, afin d'en comprendre le fonctionnement» (Considère, 2000, p. 181). Plus récemment, l'introduction de l'éducation au développement durable dans les programmes d'enseignement (Ministère de l'Éducation nationale, 2007) et la réflexion en didactique de la géographie (Vergnolle-Mainar, 2011) qui l'accompagne renforcent cette orientation vers un enseignement de la géographie à partir de situations-problèmes.

Parallèlement à ces travaux, se développe une offre didactique sur l'Internet. Les enseignants pouvant trouver une explication de la démarche d'enseignement de la géographie à l'école primaire à partir de situations-problèmes (Hugonie, 2007), voire des situations-problèmes clés en main. 
De telles propositions constituent un ensemble de prescriptions exigeantes pour les enseignants et les élèves. Elles ne sont pas sans conséquence sur ce que devrait être l'enseignement de la géographie: un enseignement problématisé. Un enseignement qui permettrait de dépasser la nomenclature et la description d'espaces singuliers pour aller vers la compréhension de faits géographiques, ceci à partir de la construction et de la résolution de problèmes.

\subsection{Les textes institutionnels: entre avancées didactiques et retours en arrière}

En France, la géographie est enseignée à partir du cycle 3 de l'école primaire pour des élèves dont l'âge est en général compris entre 8 et 11 ans. L'enseignement de cette discipline est régi par des programmes nationaux fixés par le Ministère de l'Éducation nationale (MEN).

Le programme de 2002 présentait des avancées significatives vers une géographie scolaire renouvelée. En effet, en rappelant dès l'introduction du programme que la géographie est «l'étude de l'organisation de l'espace par les sociétés» (MEN, 2002, p. 80), les auteurs proposaient une approche problématisée pour l'enseignement de la géographie à l'école élémentaire. Il était alors possible d'écrire que «la géographie scolaire propose aux élèves de l'école élémentaire des outils de lecture du monde qui ont réellement à voir avec les questions et avec les outils que les géographes peuvent mettre à sa disposition aujourd'hui» (Roumégous, 2003, p. 1).

Cette orientation est confortée par l'Inspection générale de l'Éducation nationale (IGEN) (2005) lorsqu'elle déplore que «les élèves acquièrent des connaissances ponctuelles et superficielles, dont la maîtrise n'est pas inutile, mais qui n'entrent pas dans une problématique précise et ne permettent pas la construction des notions essentielles» (p. 11).

Dans ce contexte, la publication en 2008 de nouveaux programmes pour l'école primaire marque une forte inflexion. Si l'objectif affiché pour l'enseignement de la géographie reste proche de 2002: «décrire et [de] comprendre comment les hommes vivent et aménagent leurs territoires» (MEN, 2008, p. 25), la lecture des thèmes d'étude à aborder et des commentaires qui les accompagnent mettent en évidence un profond changement qui se traduit par «un recentrage sur les niveaux scolaires nationaux, régionaux et locaux» (Chevalier, 2008, p. 32). L'entrée souvent privilégiée dans les thèmes d'étude par la description de cartes, par la présentation de données relevant de la géographie physique (reliefs, climats, cours d'eau), par la volonté de donner aux élèves des repères, risque de provoquer un retour des nomenclatures, un enseignement encyclopédique d'un savoir mort à mémoriser. Un enseignement pour l'école primaire qui privilégierait ce qui est proche et simple, l'entrée dans la complexité et la compréhension du monde, les raisonnements à partir de questionnements seraient renvoyés à plus tard. Dans ce contexte quelle place pour le problème et la problématisation?

Une tension entre deux conceptions de ce que devrait être la géographie enseignée à l'école primaire est perceptible dans ces textes institutionnels. L'une résolument novatrice met en avant un enseignement problématisé de la géographie, l'autre repose sur une conception plus traditionnelle de la géographie scolaire et de son enseignement. De ce fait, pour les enseignants, la prescription demeure relativement floue en ce qui concerne la problématisation et la place du problème en géographie. Dès lors, on peut se demander si, et comment, cette prescription est prise en compte dans les pratiques professionnelles des enseignants de l'école primaire. 


\section{Une étude empirique des pratiques d'enseignement}

Le travail réel de l'enseignant et des élèves a longtemps constitué un point aveugle des recherches sur les pratiques professionnelles des enseignants qui «ont peu porté sur l'étude empirique des pratiques d'enseignement elles-mêmes et tout particulièrement sur ce qui se passe effectivement dans la classe» (Lenoir, 2005, p. 6). Nous avons donc choisi une méthodologie fondée sur des enregistrements vidéoscopiques de séances de classes et des entretiens en autoconfrontation simple (Clot, Faïta, Fernandez et Scheller, 2000). Lors de ces entretiens, les professionnels sont confrontés à leur propre activité réalisée, ils dialoguent alors «avec l'autre, et avec eux-mêmes, se découvrant à l'image et verbalisant les conduites qu'ils observent» (Clot, 2008, p. 120). Une méthodologie qui permet d'éviter deux écueils: «ni explication externe par le chercheur, ni simple description du vécu par le sujet» (Clot, 1999, p. 137), elle autorise un croisement fructueux de deux points de vue sur la pratique professionnelle: le point de vue extrinsèque du chercheur et celui intrinsèque du sujet. Ces entretiens se distinguent, par exemple, d'entretiens postséance réalisés auprès d'enseignants de géographie du secondaire dans lesquels le chercheur leur demande de justifier leur séance, d'expliciter leurs choix (Thémines, 2004).

Le dispositif de collecte des données, identique pour chaque enseignant volontaire ${ }^{1}$, comprend l'enregistrement d'une séance de géographie proposée par l'enseignant et un entretien en autoconfrontation simple.

L'ensemble du corpus constitué par les transcriptions des neuf enregistrements vidéoscopiques et des neuf entretiens en autoconfrontation simple a fait l'objet d'une analyse de contenu à l'aide d'une grille dont les catégories d'analyse, tel qu'elles figurent dans le tableau qui suit, ont été élaborées à partir du concept de discipline scolaire (Audigier 1993; Chervel 1988).

Tableau 1: Grille d'analyse didactique des énoncés

\begin{tabular}{|l|l|l|}
\hline \multicolumn{1}{|c|}{ Catégories } & \multicolumn{2}{|c|}{ Sous-ensembles } \\
\hline \multirow{2}{*}{ A. Contenus disciplinaires } & A.1 Savoirs disciplinaires & A.1.1 Concepts; notions \\
\cline { 2 - 2 } & & A.1.2 Faits \\
\cline { 2 - 2 } & A.2 Savoir-faire disciplinaires \\
\hline \multirow{2}{*}{ B. Tâches } & B.1 Exercices spécifiques \\
\cline { 2 - 2 } & B.2 Rituels didactiques \\
\hline \multirow{2}{*}{ C. Objets } & C.1 Supports didactiques \\
\cline { 2 - 2 } & C.2 Ressources didactiques \\
\hline
\end{tabular}

Dans cette grille, ce que nous qualifions de rituels didactiques sont des exercices tellement fréquents dans les séances de géographie d'un enseignant qu'ils en deviennent de véritables rituels didactiques. C'est-à-dire des exercices que l'on fait faire aux élèves parce que c'est de la géographie et cela indépendamment des savoirs à construire, du thème de la leçon. Ils peuvent être identifiés dans le discours de l'enseignant, à partir d'expressions du type : comme on le fait habituellement en géographie. Les supports didactiques sont constitués par tout ce qui est utilisé à des fins didactiques pendant la séance, par le maître et/ou les élèves, par exemple un fond de carte à compléter. Nous considérons comme ressources didactiques, tous les éléments que le maître

1 Ce sont neuf enseignants dans des situations professionnelles variées et à différents moments de leur carrière qui ont accepté de participer à notre recherche. 
utilise pour lui, lorsqu'il prépare ou réalise sa séance, par exemple la fiche de préparation de la séance. Certains objets présents dans les séances de géographie, par exemple les manuels scolaires, peuvent être classés dans l'une ou l'autre des deux catégories en fonction de l'usage qui en est fait. Ainsi, le manuel peut être une ressource didactique quand l'enseignant l'utilise pour préparer sa séance. Il peut être un support didactique lorsqu'il est utilisé pendant la séance pour faire travailler les élèves.

Les élèves sont souvent absents des recherches sur les pratiques d'enseignement. Aussi, pour compléter notre collecte de données et pouvoir prendre en compte ce que disent les élèves de la géographie et de leurs apprentissages, un questionnaire a été adressé aux élèves de CM2 de dix classes d'écoles primaires. Au total 129 élèves ont rempli ce questionnaire. Par la suite, des entretiens semi-directifs ont été réalisés auprès d'élèves de ces dix classes. Il avait été demandé aux enseignants de ces classes de proposer trois élèves volontaires pour ces entretiens: un élève jugé bon en géographie, un élève jugé moyen et un élève jugé faible. L'ensemble des questionnaires et des 23 entretiens a été analysé.

\section{Une géographie enseignée peu problématisée}

Les résultats de notre recherche et leur analyse permettent de mettre en avant les principales caractéristiques des pratiques d'enseignement de la géographie à l'école primaire (Philippot, 2008). Se dégage alors un enseignement de la géographie peu problématisé et dans lequel les enseignants de notre corpus n'initient pas les élèves à la construction de problèmes. Cette faible problématisation se lit à travers la faible cohérence dans la construction des temps d'enseignement de la géographie et dans la persistance d'une logique d'exposition des savoirs factuels encore dominante.

\subsection{Des choix didactiques peu cohérents}

La construction d'une programmation, le choix des thèmes et de leur succession au cours de l'année relèvent de la responsabilité didactique de l'enseignant. Lors des entretiens, les enseignants ont été invités à s'exprimer sur leur programmation et le choix du thème des séances (ou de la séance) de géographie qui avaient précédé et suivi le temps d'enseignement filmé. L'analyse de leurs propos met en évidence qu'il n'y a parfois pas de programmation et que ce sont des opportunités dont se saisit l'enseignant qui donnent lieu à des temps d'enseignement de la géographie. Plus généralement, il ressort de ces propos que les thèmes sont très souvent juxtaposés sans beaucoup de liens les uns avec les autres. Constat proche de celui de l'Inspection générale de l'Éducation nationale qui souligne que l'on trouve «dans les cahiers une succession de leçons sans cohérence» (IGEN, 2005, p. 7). Cette absence d'un fil conducteur que pourrait constituer une problématique, facteur de cohérence à l'échelle de plusieurs séances, se retrouve également au niveau de chacune d'entre elles. Ainsi, les thèmes des temps d'enseignement (tableau ci-dessous), tel qu'ils sont communiqués aux élèves, restent à des niveaux de formulation très généraux. La présentation sous une forme interrogative qui pourrait être une forme élémentaire de présentation d'une problématique n'apparaît pas dans les séances enregistrées pas plus que dans les cahiers des élèves. De même, à l'oral, lors du début des séances, rien n'indique aux élèves quelle sera la perspective adoptée pour aborder ce thème. 
Tableau 2: Thèmes des temps d'enseignement

\begin{tabular}{|l|l|l|}
\hline Enseignant & Thème de la séance & Classe $^{\mathbf{2}}$ \\
\hline Mme Har. & Pays riches / pays pauvres & CM1 / CM2 \\
\hline Mme Jou. & Les villes d'Europe & CM2 \\
\hline Mme Liv. & $\begin{array}{l}\text { Situer des monuments de la ville sur un } \\
\text { plan en utilisant les TICE }\end{array}$ & CE2 / CM1 \\
\hline Mlle Mat. & Pays riches / pays pauvres & CM2 \\
\hline Mme Sou. & Les DOM TOM & CE2 / CM1 / CM2 \\
\hline M. Dej. & Les zones d'influence en France & CM1 / CM2 \\
\hline M. Fran. & Les climats de la France & CM2 \\
\hline M. Rom. & L'agriculture française & CM2 \\
\hline M. Thi. & L'Europe & CM2 \\
\hline
\end{tabular}

La problématique didactique, qu'il convient de distinguer de la problématique scientifique, sert à fonder les choix de l'enseignant, elle propose «un cheminement intellectuel roboratif, elle implique la recherche de réponses, elle sollicite l'argumentation» (Maréchal, 1994, p. 248). En d'autres termes, l'élaboration d'une problématique didactique - la problématisation - confère au temps d'enseignement sa cohérence, elle constitue le fil conducteur à la fois pour l'enseignant et pour les élèves. En l'absence d'une telle problématisation, d'ailleurs peu présente dans le programme d'enseignement de la géographie de 2008, les temps d'enseignements de la géographie apparaissent comme des constructions singulières et contingentes juxtaposées les unes aux autres qui s'inscrivent dans une logique d'exposition du savoir.

\subsection{La logique d'exposition d'un savoir factuel}

Les neuf séances analysées donnent à voir un enseignement de la géographie où les données factuelles, parfois de sens commun, sont largement dominantes par rapport à la mobilisation de notions et concepts géographiques. Une telle prégnance des données factuelles n'est pas sans effet sur les apprentissages des élèves. Ainsi, lorsque nous avons interrogé des élèves en fin de cycle 3 en leur proposant de répondre à la question: «Qu'as-tu appris en géographie depuis le CE2? Donne des exemples», ceux-ci ont répondu très majoritairement en indiquant une liste d'objets, des «choses», assez hétérogènes. Par exemple, comme l'écrit cet élève sur son questionnaire:

«Depuis le CE2 j'ai appris énormément de choses, dont: on a appris les 4 points cardinaux, les pays frontaliers de la France, le globe terrestre, les continents et les océans, les zones climatiques, les zones froides du globe, les richesses du désert, la répartition de la population sur la terre, le milieu tempéré, les montagnes françaises, l'Europe, l'Union européenne, le littoral français, départements et régions. C'est à peu près tout ce qu'on a appris, il manque des choses.» (Extrait questionnaire)

2 Les cours élémentaire deuxième année (CE2), cours moyen première année (CM1) et cours moyen deuxième année (CM2) constituent le troisième et dernier cycle de l'école primaire française. Le CE2 accueille des élèves de 8-9 ans, le CM1 des élèves de 9-10 ans et le CM2 des élèves de 10-11 ans. 
Cet enseignement que l'on peut qualifier de factuel prend place dans des dispositifs qui laissent peu de place à l'élève considéré comme un sujet en situation d'apprendre. En effet, un mode de travail dominant du travail en classe se dégage, celui d'un enseignement essentiellement oral sous forme de questions/réponses, qualifié de cours dialogué. Fréquemment sollicités par leurs enseignants pour décrire un document, lire un extrait de texte ou répondre à une question, les élèves disposent d'un temps de parole limité, en général deux ou trois secondes, pour formuler une réponse. Cela laisse peu d'espace à la réflexion, à la construction d'un raisonnement. Observer, décrire, nommer sont les opérations intellectuelles les plus mobilisées. Raisonner, argumenter, résoudre des problèmes sont autant d'activités intellectuelles rarement sollicitées.

Dans ces conditions, le temps d'enseignement de la géographie reste pour l'essentiel un temps magistro-centré. Le savoir est considéré comme un déjà-là que l'enseignant révèle aux élèves. La recherche de la parole des élèves, leur mise en activité à partir de divers documents est un habillage qui participe à la mise en scène d'un savoir qui est exposé aux élèves. Ce modèle traditionnel de l'enseignement de la géographie (Audigier et Tutiaux-Guillon, 2004) traverse les années et résiste aux évolutions épistémologiques et à celles des prescriptions. Un tel modèle qui accorde peu de place à la problématisation des savoirs, quand il ne s'y oppose pas, s'alimente pour l'essentiel aux conceptions qu'ont les enseignants de la géographie, de la façon dont les élèves apprennent et aux difficultés de tous ordres qu'ils rencontrent lorsqu'il s'agit d'enseigner cette discipline.

\subsection{La géographie ou la «réalité» du monde donnée directement à voir}

Enseigner de la géographie, c'est enseigner «ce qui est proche des élèves, ce qui est réputé plus concret» (Audigier, 1999, p. 402), telle est une représentation commune chez les enseignants du primaire que l'on retrouve, par exemple, dans les propos de cet enseignant:

«Alors la géographie en primaire, c'est l'étude du réel. [...]. Il suffit de regarder dehors et on peut, là on est dans le concret, on fait une étude de quelque chose de concret et puis de quelque chose là encore une fois qui les touche vraiment directement.» (Extrait entretien M. Rom.)

Cette représentation n'est pas sans conséquence didactique; elle induit des pratiques d'enseignement qui oublient les langages, les problématiques qui construisent les savoirs. Ces pratiques livrent donc le monde aux élèves «dans sa complétude et de façon transparente [...], c'est-à-dire sans médiation» (Clerc, 2002, p. 48), notamment en mobilisant des supports didactiques de type iconographique, considérés comme des «fragments du réel» (Ibid.). Cette assimilation de ces supports, et au-delà, de l'enseignement de la géographie, à la réalité du monde, au concret, peut développer chez les élèves l'illusion d'être de plain-pied dans le monde (Orain, 2009). Dès lors, dans la classe, la mise en scène du savoir et sa révélation prennent le pas sur la construction de celui-ci. La problématisation des savoirs n'est pas nécessaire, il suffit d'observer et de décrire le monde, ce qui conforte la logique de l'enseignement factuel.

Dans les classes, la distance est donc importante entre un idéal didactique que serait un enseignement problématisé de la géographie et la géographie enseignée. Nos résultats mettent en évidence que l'enseignant est beaucoup plus porté sur le versant enseignement, entendu ici comme transmission de connaissances factuelles, que sur le versant apprentissage des élèves, 
avec comme postulat, probablement implicite pour beaucoup d'enseignants, qu'il suffirait que l'enseignant enseigne pour que les élèves apprennent, surtout s'ils sont actifs, c'est-à-dire s'ils répondent bien aux sollicitations de l'enseignant. Cette activité des élèves n'est que superficielle et renvoie au fait que l'enseignant «s'efforce simplement de faire redécouvrir le savoir par les élèves de manière active» (Astolfi, 2008, p. 155). Dès lors, la problématisation des savoirs, la construction de problèmes à proposer aux élèves ne semblent pas être au cœur de leurs préoccupations lorsqu'ils conçoivent et mettent en œuvre leur enseignement. De plus, au regard des difficultés d'ordre identitaire, épistémique et pragmatique que ces enseignants expriment à propos de l'enseignement de la géographie, une approche problématisée de cet enseignement les confronterait à un triple défi.

\section{La problématisation: un triple défi pour l'enseignant}

S'engager sur la voie d'un enseignement problématisé, proposer aux élèves des problèmes à résoudre, voire à construire, suppose pour l'enseignant de se démarquer du modèle traditionnel, de sortir des sentiers battus de l'enseignement de la géographie: «commencer à questionner est un saut dans l'inconnu, une prise de risques» (Étévé, 2005, p. 126). Dans cette voie l'enseignant doit alors relever un triple défi.

\subsection{Un défi épistémologique}

La géographie enseignée témoigne de la difficile connaissance et maîtrise de l'épistémologie de la géographie. Sans pour autant attendre des enseignants du primaire une maîtrise épistémologique qui serait celle des enseignants spécialistes du secondaire, on peut estimer que la connaissance et la maîtrise des matrices disciplinaires, c'est-à-dire du principe d'intelligibilité, du paradigme qui organise «la totalité des contenus en un ensemble cohérent» (Develay, 1995, p. 27) pour une discipline scolaire, sont des conditions nécessaires au développement d'un enseignement problématisé.

De plus, pour l'enseignant, s'engager vers ce type d'enseignement suppose également une nouvelle conception du savoir, non plus comme «un texte qui énonce des vérités, comme un produit tout élaboré à transmettre» (Astolfi, 2008, p. 155), mais comme une construction dans le cadre d'une discipline: la géographie. Cet engagement suppose aussi de concevoir différemment le rapport des élèves aux savoirs disciplinaires et ne pas considérer les processus d'apprentissage nécessaire à son appropriation comme allant de soi.

Enfin, il suppose de répondre à la question de la nature du problème à construire ou à faire construire. Doit-on, dans l'enseignement de la géographie à l'école primaire, prendre appui sur la logique rationaliste de Bachelard, marquée par «l'idée de rupture, d'obstacle épistémologique» (Fabre, 2005a, p. 62) ou sur la logique pragmatique de Dewey qui pose une continuité entre la vie quotidienne et le savoir scientifique, «toute vie est résolution de problème» (Ibid.)? Au vu de nos résultats, on peut avancer que la logique rationaliste, celle de la rupture épistémologique, semble plus difficile à mobiliser et à mettre en œuvre par les enseignants du primaire. En effet, lorsque, par exemple, les enseignants cherchent à recueillir les représentations initiales des élèves, ce n'est pas pour les analyser et dégager des objectifs ou obstacles à dépasser pour construire un nouveau savoir à partir de problèmes, mais pour partir des élèves, pour susciter leur intérêt au début d'une séance. Il semble possible d'avancer que la logique pragmatique et la théorie de 
l'enquête de Dewey (Fabre, 2005a) offrent une perspective plus en rapport avec des pratiques d'enseignement de la géographie dans lesquelles l'expérience personnelle et quotidienne des élèves est fréquemment convoquée. Dans la mesure où ces situations quotidiennes «ne sont pas complètement indéterminées» et à partir du peu qu'ils savent, les élèves pourraient «émettre des hypothèses et les tester» (Ibid., 2005a, p. 54). La posture de l'élève pourrait alors changer, il ne lui serait plus demandé de «savoir-répondre», mais de «savoir-questionner» (Ibid.). Une logique qui engage «du côté des pédagogies du projet» (Ibid., p. 65), forme pédagogique fréquemment mobilisée par les enseignants de l'école primaire et qui pourrait dès lors constituer un point de rencontre entre leurs pratiques d'enseignement et l'approche par problème, pour peu qu'ils soient aidés dans ce cheminement.

\subsection{Un défi didactique}

L'élaboration de situations-problèmes dans la logique rationaliste de Bachelard ou bien encore la problématisation dans le cadre d'une pédagogie de projet nécessitent toutes deux une connaissance du contenu à enseigner (Shulman, 1987, 2004). Or, au vu de nos résultats, cette connaissance s'avère être relativement modeste. Dans ces conditions, cette élaboration de problèmes par l'enseignant, mais aussi la conduite en situation professionnelle de l'activité de problématisation par les élèves, demeurent problématiques. L'analyse des différentes transcriptions des enregistrements vidéoscopiques réalisés montre à quel point les interactions didactiques et leur régulation restent un défi pour l'enseignant.

\subsection{Un défi éthique}

En dernier lieu, l'introduction du problème en classen'est pas sans soulever des questions relevant de l'éthique professionnelle. On peut se demander si la logique du problème qui suppose analyse, recherche de réponses, argumentation, débat, et participe d'une certaine façon au développement de l'esprit critique de l'élève n'entre pas en conflit avec une des finalités de l'enseignement de la géographie à l'école primaire qui est de construire une vision commune, partagée par tous les élèves, du territoire national et de sa place en Europe et dans le Monde. En effet, la géographie scolaire en tant que discipline scolaire vise la construction d'un référent consensuel, c'est-à-dire «un monde qui est accepté par tous, gommant les débats, les oppositions qui sont ceux des hommes et des sociétés lorsqu'ils parlent d'eux-mêmes, de leurs visions du monde, de leurs mémoires, de leurs territoires...» (Audigier, 1993, p. 161). Elle tait les enjeux éthiques et politiques inhérents à la production des savoirs qu'elle enseigne. Dès lors, on peut se demander si l'entrée par le problème ne risque pas de faire réapparaître dans la classe ce que la discipline scolaire cherche à gommer, plaçant alors l'enseignant en situation difficile.

\section{Conclusion}

Dans de telles conditions, on peut comprendre les raisons pour lesquelles une approche problématisée de l'enseignement de la géographie reste pour l'essentiel de l'ordre de l'idéal. Pour les enseignants, les changements qu'elle suppose rendent nécessaire la rupture d'avec les certitudes que leur offre le cadre de la discipline scolaire. Prendre en compte cette approche, la mettre en 
œuvre dans le quotidien de la classe supposeraient une prise de risque de la part de l'enseignant. Prise de risque qu'il n'est pas certain que les enseignants soient prêts à assumer dans les contextes professionnels actuels.

De tels constats conduisent à avancer quelques perspectives quant à la formation initiale et continue des enseignants de l'école primaire dans le contexte français. Actuellement les enseignants de l'école primaire sont recrutés au niveau master $(\mathrm{Bac}+5)$ après des études universitaires souvent effectuées dans des licences spécialisées. De ce fait, ces étudiants, après la fin de leurs études secondaires, sont coupés d'une partie importante des disciplines scolaires qu'ils retrouveront, pour l'essentiel, une fois devenus enseignants. On peut donc penser qu'une formation universitaire qui permettrait, pendant les trois années de licence, de maintenir ces liens avec la majorité des disciplines à enseigner à l'école primaire serait susceptible de favoriser l'acquisition des matrices disciplinaires (Develay, 1992). Dans le champ de la formation continue, il semble nécessaire d'envisager un accompagnement professionnel des enseignants leur permettant de s'engager dans la voie d'un enseignement problématisé de la géographie. En lien avec cet accompagnement qui peut passer par le développement de groupes de formation à et par la recherche, la mise à disposition d'un outillage didactique qui puisse véritablement servir de ressource aux enseignants apparaît comme indispensable. C'est à ces conditions que l'enseignement problématisé de la géographie à l'école primaire ne restera pas cantonné aux marges des pratiques d'enseignement et ne relèvera pas uniquement d'enseignants novateurs.

\section{Références}

Astolfi, J.-P. (2008). La saveur des savoirs. Disciplines et plaisir d'apprendre. Paris: ESF éditeur.

Audigier, F. (1991). Recherches en didactique de l'histoire, de la géographie, des sciences sociales. Problèmes et Problématiques. In F. Audigier et G. Baillat (dir.), Analyser et gérer les situations d'enseignement-apprentissage (p. 11-18). Paris: Institut national de Recherche pédagogique.

Audigier, F. (1993). Les représentations que les élèves ont de l'histoire et de la géographie. À la recherche des modèles disciplinaires, entre leur définition par l'institution et leur appropriation par les élèves. Thèse en didactique des disciplines, Université Paris 7.

Audigier, F. (1999). Les représentations de la géographie dans l'enseignement primaire en France. Habitat commun, voisinages et distances. Cahiers de géographie du Québec, 120, 395-412.

Audigier, F. et Tutiaux-Guillon, N. (dir.) (2004). Regards sur l'histoire, la géographie et l'éducation civique à l'école élémentaire. Paris: Institut national de Recherche pédagogique.

Bachelard, G. (1970). La formation de l'esprit scientifique. Paris: Vrin.

Baldner, J.-M., Clary, M. et Élissalde, B. (dir.) (1995). Histoire, géographie et éducation civique dans les cycles à l'école élémentaire. Éléments d'une recherche. Paris: Institut national de Recherche pédagogique.

Benoît, J.-P. (2007). La problématisation dans l'enseignement en France entre 2000 et 2006. Actes du Congrès international AREF 2007. Strasbourg.

Bouchut, A. (2006). Enseigner la ville à l'école élémentaire: quels dispositifs d'apprentissage pour appréhender l'espace urbain au cycle III? L'information géographique, 70, 77-104.

Cariou, J.-Y. (2009). Former l'esprit scientifique en privilégiant l'initiative des élèves dans une démarche s'appuyant sur l'épistémologie et l'histoire des sciences. Thèse de doctorat. Genève. Document téléaccessible à l'adresse $<$ http://tel.archives-ouvertes.fr/docs/00/52/11/74/PDF/THESE_JYC_.pdf $>$. 
Chervel, A. (1988). L'histoire des disciplines scolaires. Réflexions sur un domaine de recherche. Histoire de l'éducation, $38,59-119$.

Chevalier, J.-P. (2008). Enseigner la France en géographie aux jeunes écoliers (1788-2008). L’information géographique, 3, 20-33.

Clerc, P. (2002). La culture scolaire en géographie. Le monde dans la classe. Rennes: Presses universitaires de Rennes.

Clot, Y. (1999). La fonction psychologique du travail. Paris: Presses universitaires de France.

Clot, Y. (2008). Travail et pouvoir d'agir. Paris: Presses universitaires de France.

Clot, Y., Faïta, D., Fernandez, G. et Scheller, L. (2000). Entretiens en autoconfrontation croisée: une méthode en clinique de l'activité. Pistes, Vol.1, 1, 1-7. Document téléaccessible à l'adresse $<\mathrm{http}: / / w w w . p i s t e s . u q a m . c a / v 2 n 1 /$ sommaire.html>.

Considère, S. (2000). Raisonner en géographie au cycle 3 de l'école élémentaire. Hommes et Terres du Nord, 3, 180-188.

Daniellou, F. (2002). Le travail des prescriptions. Actes du XXXVII congrès de la SEF. Aix-en-Provence.

Demers, S., Lefrançois, D. et Éthier, M.-A. (2010). Un aperçu des écrits publiés en français et en anglais depuis 1990 à propos de recherches en didactique sur le développement de la pensée historique au primaire. In J.-F. Cardin, A. Meunier et M.-A. Éthier (dir.), Histoire, musées et éducation à la citoyenneté : recherches récentes. (p. 213-245). Montréal : Multimondes.

Develay, M. (1992). De l'apprentissage à l'enseignement. Pour une épistémologie scolaire. Paris: ESF éditeur.

Develay, M. (1995). Savoirs scolaires et didactique des disciplines. Paris: ESF éditeur.

Dictionnaire Le Robert (1992). Dictionnaire historique de la langue française. Paris: Dictionnaires Le Robert.

Étévé, C. (2005). Problématisation: problématisation, dé-problématisation et re-problématisation. Recherche et Formation, 48, 119-134.

Fabre, M. (1997). Pensée pédagogique et modèles philosophiques: le cas de la situation-problème. Revue française de pédagogie, 120, 49-58.

Fabre, M. (2005a). Deux sources de l'épistémologie des problèmes: Dewey et Bachelard. Les Sciences de l'éducation - Pour l'ère nouvelle, 38(3), 53-67.

Fabre, M. (2005b) Introduction du numéro thématique, La problématisation: approches épistémologiques. Les Sciences de l'éducation - Pour l'ère nouvelle, 38(3), 7-10.

Gérin-Grataloup, A.-M., Solonel, M. et Tutiaux-Guillon, N. (1996). Situations-problèmes et situations scolaires en histoire-géographie. Revue française de pédagogie, 106, 25-37.

Hugonie, G. (2007). Conférence géographie école primaire. 24 octobre. Synthèse réalisée par le Groupe départemental Géographie du Finistère. Document téléaccessible à l'adresse <http://www.ien-landivisiau.ac-rennes.fr/ geographie/conference\%20hugonie.pdf>.

Inspection générale de l'Éducation nationale, (2005). Sciences expérimentales et technologie, histoire et géographie. Leur enseignement au cycle 3 de l'école primaire. Rapport № 2005-112.

Lautier, N. (2006). Introduction. In V. Haas (dir.), Les savoirs du quotidien. Transmissions, Appropriations, Représentations (p. 71-75). Rennes: Presses universitaires de Rennes.

Lenoir, Y. (dir.), (2005). Les pratiques enseignantes: analyse des données empiriques. Les dossiers des sciences de l'éducation, 14.

Le Roux, A. (1997). Didactique de la géographie. Caen: Presses universitaires de Caen.

Le Roux, A. (2004). Enseigner l'histoire-géographie par le problème? Paris: L'Harmattan. 
Maréchal, J. (1994). Démarche pour préparer, réaliser, analyser une leçon de géographie. In P. Desplanques (dir.), $L a$ géographie en collège et lycée (p. 246-263). Paris: Hachette Éducation.

Masson, M. (1994). Vous avez dit géographies. Didactique d'une géographie plurielle. Paris: Armand Colin.

Ministère de l'Éducation nationale (2002). Horaires et programmes d'enseignement de l'école primaire. Bulletin officiel du Ministère de l'Éducation nationale et du Ministère de la Recherche, $\mathrm{n}^{\circ} 1$ Hors série, 14 février.

Ministère de l'Éducation nationale, (2007). Éducation au développement durable. Bulletin officiel du Ministère de l'Éducation nationale et du Ministère de la Recherche, $\mathrm{n}^{\circ} 14,5$ avril.

Ministère de l'Éducation nationale (2008). Horaires et programmes d'enseignement de l'école primaire. Bulletin officiel du Ministère de l'Éducation nationale et du Ministère de la Recherche, $\mathrm{n}^{\circ} 3$ Hors série, 13 juin.

Orain, O. (2009). De plain-pied dans le monde. Écriture et réalisme dans la géographie française au XXe siècle. Paris: L'Harmattan.

Orange, C. (2005). Problématisation et conceptualisation en sciences et dans les apprentissages scientifiques. Les Sciences de l'éducation - Pour l'ère nouvelle, 3(3), 68-94.

Partoune, C. (2002). La pédagogie par situations-problèmes. Puzzle, Université de Liège. Document téléaccessible à l'adresse <http://www.lmg.ulg.ac.be/spip/IMG/sit_pbl_texte.pdf >. Consulté le 18 novembre 2011.

Philippot, T. (2008). La professionnalité des enseignants de l'école primaire: les savoirs et les pratiques. Thèse de doctorat, Reims: Université Reims Champagne-Ardenne.

Rey, B. (2005). Peut-on enseigner la problématisation? Recherche et Formation, 48, 91-105.

Roumégous, M. (2003). «Découvrir le monde»: quelle géographie veut-on faire apprendre à l'école élémentaire? Association française pour le développement de la géographie. Document téléaccessible à l'adresse $<\mathrm{http}: / / \mathrm{www}$. afdg.org/spip/article.php3?id_article=1>.

Saujat, F., Amigues, R. et Faïta, D. (2007). Les compétences pour enseigner comme ressources et produits de l'activité du professeur. In L. Talbot et M. Bru (dir.), Des compétences pour enseigner. Entre objets sociaux et objets de recherche (p. 183-196). Rennes: Presses universitaires de Rennes.

Shulman, L.S. (1987). Knowledge and teaching: foundations of the new reform. In L. S. Shulman (2004) Teaching as community property (p. 84-113). The Carnegie Foundation for the Advancement of Teaching.

Tardif, M. et Lessard, C. (1999). Le travail enseignant au quotidien. Expérience, interactions humaines et dilemmes professionnels. Bruxelles: De Boeck Université.

Thémines, J.-F. (2004). Quatre conceptions de la géographie scolaire: un modèle interprétatif de pratiques d'enseignement de la géographie. Cybergeo : European Journal of Geography, Épistémologie, Histoire de la géographie, Didactique. Document téléaccessible à l'adresse <http://cybergeo.revues.org/4325> .

Vergnolle-Mainar, C. (2011). La géographie dans l'enseignement. Une discipline en dialogue. Rennes: Presses universitaires de Rennes. 